\title{
$\mathrm{CH}$ 간 \\ Swiss Science Concentrates \\ A CHIMIA Column \\ Terminal-Selective Functionalization of Alkyl Chains by Regioconvergent Cross-Coupling
}

S. Dupuy, K.-F. Zhang, A.-S. Goutierre, and O. Baudoin*, Angew. Chem. Int. Ed. 2016, 55, 14793. University of Basel

The direct functionalization of hydrocarbon chains obtained from cheap and abundant feedstocks provides economical access to organic intermediates rich in tetrahedral carbon atoms. In this paper, Baudoin and coworkers describe a stepeconomical approach that enables $\mathrm{C}-\mathrm{C}$ bond formation at the terminal position of linear alkanes. They show that secondary alkyl bromides undergo in situ conversion into alkyl zinc bromides followed by regioconvergent Negishi cross-coupling with aryl or alkenyl triflates. Mixtures of secondary alkyl bromides are prepared by standard bromination of linear alkanes. Using a suitable Yha p ho s p h i n e ligand favoring $\mathrm{Pd}$ migration allows selective formation of the linear arylation product in only two steps.

\section{Development of a Photoactivatable Phosphine Probe for Induction of Intracellular Reductive Stress with Single-Cell Precision}

A. Tirla and P. Rivera-Fuentes*, Angew. Chem. Int. Ed. 2016, 55, 14709. ETH Zurich

Disruption of redox homeostasis that produces excessively reducing environments has been associated with numerous pathologies, including cancer, inflammation, metabolic disorders, and neurodegenerative diseases. Tirla and Rivera-Fuentes report photoactivatable phosphines that induce intracellular reductive stress. The design of these probes takes advantage of the conjugate addition of trialkylphosphines to carbocyanine dyes, which can be reverted photochemically to produce the trialkylphosphine and a fluorescent reporter. The probes readily permeate the mammalian

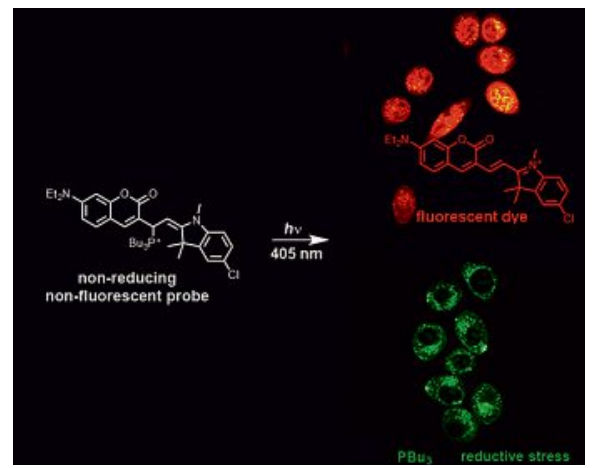
plasma membrane and can be activated in live cells. Upon irradiation, the released trialkylphosphine induces reductive stress, which leads to the formation of thioflavin-positive intracellular protein aggregates.

\section{Silica-Supported Cu Nanoparticle Catalysts for Alkyne Semihydrogenation: Effect of Ligands on Rates and Selectivity}

A. Fedorov*, H.-J. Liu, H.-K. Lo, and C. Copéret, J. Am. Chem. Soc. 2016, 138, 16502. ETH Zurich

The stereoselective semihydrogenation of alkynes to $Z$-alkenes is a key step in the synthesis of vitamins and many natural products. Typically, this reaction implies the use of expensive and rare palladium and toxic lead additive (Lindlar catalyst). Furthermore, it may suffer from limitations, such as a narrow substrate scope, over-hydrogenation, $Z / E$ isomerization or double bond migration. Fedorov, Copéret and coworkers demonstrate the use of narrowly-dispersed, silica-supported $\mathrm{Cu}$ nanoparticles as very active catalysts for the hydrogenation of a broad range of alkynes. Optimal ligands and reaction conditions were identified by a high throughput screening approach. The best catalyst $\left(\mathrm{Cy}{ }_{3} \mathrm{P}-\mathrm{Cu} / \mathrm{SiO}_{2-700}\right)$ allows the highly chemo- and stereoselective preparation of Z-olefins, thus providing an efficient and cheap alternative to the Lindlar catalyst.

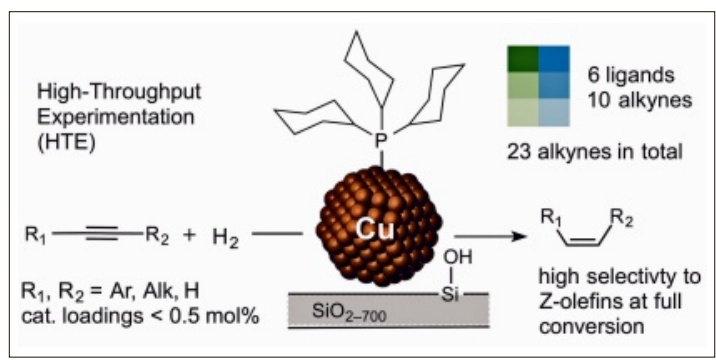

\section{Synthesis of Triazolylidene Nickel Complexes and their Catalytic Application in Selective Aldehyde Hydrosilylation}

Y. Wei, S.-X. Liu, H. Mueller-Bunz, and M. Albrecht*, ACS Catal. 2016, 6, 8192. University of Bern

Reduction of carbonyl compounds is a fundamental process in organic synthesis. In catalytic hydrosilylation, both the reduction of carbonyl functionality and protection of the resulting alcohol in the form of silylethers can be accomplished in a single step with high atom economy. Albrecht and coworkers have synthesized a set of triazolylidene-based nickel(II) complexes for this purpose. They demonstrated that the choice of substituents on the triazolylidene ligand is critical for the catalytic activity of the metal complexes. In particular, a pyridyl-derived complex reached

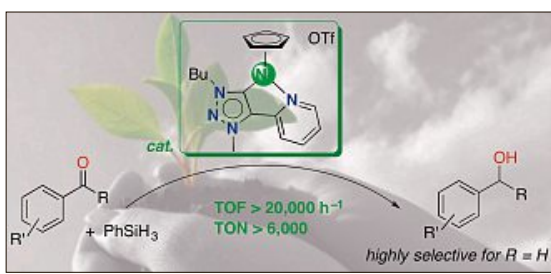
turnover frequencies of $>20000 \mathrm{~h}^{-1}$ with good catalyst stability. In addition, this catalyst allows the selective hydrosilylation of aldehydes in the presence of ketones. 